\title{
Therapeutic Benefit in Allergic Dermatitis Derived from the Inhibitory Effect of Byakkokaninjinto on the Migration of Plasmacytoid Dendritic Cells
}

\author{
Takeshi Yamamoto D, Yue Zhang, Ai Kigasawa, Shusaku Hayashi, and Makoto Kadowaki \\ Division of Gastrointestinal Pathophysiology, Institute of Natural Medicine, University of Toyama, 2630 Sugitani, \\ Toyama 930-0194, Japan \\ Correspondence should be addressed to Takeshi Yamamoto; ty@inm.u-toyama.ac.jp
}

Received 27 May 2020; Revised 7 September 2020; Accepted 23 September 2020; Published 23 October 2020

Academic Editor: XiuMin Li

Copyright (c) 2020 Takeshi Yamamoto et al. This is an open access article distributed under the Creative Commons Attribution License, which permits unrestricted use, distribution, and reproduction in any medium, provided the original work is properly cited.

\begin{abstract}
Dendritic cells (DCs) are well known to be essential immunocytes involved in innate and adaptive immunity. DCs are classified as conventional dendritic cells (cDCs) and plasmacytoid dendritic cells (pDCs). Recently, the accumulation of pDCs in inflamed tissues and lymphoid tissues has been considered to be a possible contributing factor in the development of immunological diseases, but little is known about the pathophysiological roles of pDCs in immunological diseases. To date, many studies have demonstrated that many kinds of Kampo formulas can regulate immunological reactions in human immune diseases. Thus, we screened Kampo formulas to identify an agent that inhibits pDC migration. Furthermore, we investigated the therapeutic effects of these formulas on a murine DNFB-induced allergic contact dermatitis model. Bone marrow-derived pDCs (BMpDCs) were derived from the bone marrow cells of $\mathrm{BALB} / \mathrm{c}$ mice in a culture medium with Flt3 ligand. The effects of Kampo formulas on $\mathrm{BMpDC}$ migration were evaluated by assessing the number, velocity, and directionality of BMpDCs chemotaxing toward the more concentrated side of a chemokine (C-C motif) ligand 21 (CCL21) gradient. The Kampo formulas that exerted inhibitory effects on pDC migration were orally administered to DNFB-induced allergic contact dermatitis model mice. Byakkokaninjinto reduced the number of migrated BMpDCs and suppressed the velocity and directionality of BMpDC migration in a chemotaxis assay. Gypsum Fibrosum and Ginseng Radix, which are components of byakkokaninjinto, obviously suppressed the velocity of BMpDC migration. Furthermore, Gypsum Fibrosum significantly suppressed the directionality of BMpDC migration. In DNFB-induced allergic contact dermatitis model mice, byakkokaninjinto markedly abrogated ear swelling in late-phase allergic reactions. In conclusions, byakkokaninjinto, which has an inhibitory effect on pDC migration, was able to prevent the occurrence of allergic contact dermatitis, suggesting that pDCs were involved in the onset of allergic contact dermatitis in the mouse model. Therefore, byakkokaninjinto is anticipated to be a therapeutic agent for disorders related to pDC migration.
\end{abstract}

\section{Introduction}

Dendritic cells (DCs) play a critical role in immune regulation. In particular, DCs possess a strong ability to present antigens to $\mathrm{CD}^{+}{ }^{+} \mathrm{T}$ cells. DCs, as versatile immunocytes, play roles in the induction of T-cell activation at the beginning of an immune response and in immune tolerance. During these processes, DCs need to migrate to organs and tissues to exert their functions. The migration of DCs is induced by chemokines and depends on chemokine concentration gradients [1]. In general, DCs have been divided into conventional dendritic cells (cDCs) and plasmacytoid dendritic cells (pDCs). The bone marrow is the primary site of $\mathrm{pDC}$ production, and $\mathrm{pDCs}$ migrate through the circulation to the thymus, secondary lymphoid organs, and peripheral tissues. In the normal state, pDCs rarely exist in peripheral tissues, but once viral infection occurs, pDCs accumulate in infected sites and rapidly secrete massive amounts of type 1 IFN. pDCs are activated by viruses; subsequently, they extend, form dendrites, and express the 
costimulators MHCII, CD40, CD80, and CD86 for antigen presentation [2]. Consequently, the migration of pDCs is important in facilitating the immune functions of these cells.

Chemokine (C-C motif) ligand 19 (CCL19) and chemokine (C-C motif) ligand 21 (CCL21), as ligands of C-C chemokine receptor type 7 (CCR7), are highly expressed in secondary lymphoid tissues [3]. The numbers of $120 \mathrm{Gr}^{+}$ $\mathrm{B} 220^{\mathrm{int}} \mathrm{CD} 11 \mathrm{c}^{\mathrm{int}} \mathrm{pDCs}$ are decreased in the peripheral lymph nodes and mesenteric lymph nodes of CCR7-deficient mice [4]. Thus, CCR7 is required for pDC migration to the lymph nodes both under steady-state conditions and during viral infections [5].

pDCs have been reportedly related to inflammatory skin disorders [6]. Infiltrated pDCs have been found in the skin of systemic sclerosis patients, whereas pDCs in the peripheral blood have been found to be decreased, and depletion of $\mathrm{B} 220^{+}$PDCA- $1^{+}$pDCs reduces skin thickness in a skin fibrosis model [7]. Similarly, pDCs are increased, and pDCs accumulate in inflamed sites in patients with contact dermatitis $[8,9]$. Otherwise, it has been reported that pDCs are increased in the lesional skin of atopic dermatitis patients [8-10]. Therefore, pDCs play essential roles in the pathogenic mechanism and defense mechanisms of many inflammatory skin disorders, including atopic dermatitis.

Kampo formulas have been used according to individual situations. Many Kampo formulas target the immune system, and their therapeutic effects on immunological diseases have been demonstrated in clinical studies [11, 12]. However, the precise mechanisms of Kampo formulas are not well understood. In addition, little is known about the effects of Kampo formulas on the function of DCs, a target for immunological diseases, even though DCs play a key role in immune responses.

Byakkokaninjinto, a traditional Japanese Kampo formula, has been frequently used in Japan and originates from the classical Chinese records of 'Shang Han Lun' and 'Jin Gui Yao Lue'. Byakkokaninjinto is composed of Gypsum Fibrosum, Ginseng Radix, Glycyrrhizae Radix, Anemarrhenae Rhizoma, and Oryzae Fructus (Table 1). Gypsum Fibrosum, as the basic component of byakkokaninjinto, is composed of calcium sulfate, and Kampo formulas containing Gypsum Fibrosum are used for their suppressive effects on heat and inflammation [13]. Anemarrhenae Rhizoma has anti-inflammatory effects [14]. Ginseng Radix has been used as an antiviral drug [15]. Therefore, byakkokaninjinto is widely applicable for the treatment of many disorders, such as oral dryness caused by diabetes, dermatitis, eczema, urticaria, pneumonia, and the common cold [16]. Dermatitis is one of the indications of byakkokaninjinto, and the efficacy of byakkokaninjinto in clinically curing atopic dermatitis and allergic contact dermatitis has been reported $[16,17]$. In addition, it has been reported that byakkokaninjinto induces therapeutic effects on spontaneous atopic dermatitis-like skin lesions in NC mice [18] and IgE-mediated triphasic skin reaction [19]. However, the antiinflammatory mechanism underlying the effects of byakkokaninjinto on these dermatitis remains uncertain.

In this study, we investigated the effects of 86 Kampo formulas on $\mathrm{pDC}$ migration and demonstrated that byakkokaninjinto exerted therapeutic effects on an DNFBinduced allergic contact dermatitis model by inhibiting pDC migration.

\section{Materials and Methods}

2.1. Mice. Male BALB/c mice (6-10 weeks old) were purchased from Japan SLC (Shizuoka, Japan). All mice were housed under standard vivarium conditions $\left(23.5 \pm 0.5^{\circ} \mathrm{C}\right.$, 12-hour light/dark cycle, and food and water provided ad libitum). This study was performed in strict accordance with the recommendations of the Guide for the Care and Use of Laboratory Animals by the National Institutes of Health. The Animal Experiment Committee at the University of Toyama approved all the animal care procedures and experiments (authorization numbers A2012 INM4, A2015 INM-3, and A2018 INM-4).

\subsection{Preparation of Kampo Formulas and Each Component's} Extracts. Kampo formula extracts and component extracts were provided as dried powders by the Joint Usage/Research Center for Science-Based Natural Medicine, Institute of Natural Medicine, University of Toyama, and the Knowledge Cluster Initiative Program (Second Stage) of the Ministry of Education, Culture, Sports, Science and Technology of Japan. Each herbal extract was obtained using a standard method. In brief, each formula and each herbal component was extracted in water at $100^{\circ} \mathrm{C}$ for $50 \mathrm{~min}$, evaporated under reduced pressure, and freeze-dried to obtain a powder extract. The 86 Kampo formula extracts studied are shown in Table 2. Detailed information about the herbal components of the 86 Kampo formula extracts and detailed information of herbal component extracts are shown in Supplemental Tables 1 and 2.

For a mouse allergic contact dermatitis model study, Byakkokaninjinto (TJ-34) was purchased from Tsumura Co. (Tokyo, Japan) as a dried powder with the 3D-HPLC data (Supplemental Figure 1) and was evaluated as a therapeutic drug for the symptoms of DNFB-induced allergic contact dermatitis.

2.3. BMpDC Generation. Bone marrow-derived plasmacytoid dendritic cells (BMpDCs) were generated from bone marrow cells according to a method described previously [20]. Briefly, bone marrow cells were collected from the femur and tibia of male BALB/c mice (6-10 weeks old) and incubated in RPMI 1640 medium (Wako, Osaka, Japan) supplemented with $10 \%$ FBS (Equitech-Bio, Kerrville, TX, USA), $55 \mu \mathrm{m}$ 2-mercaptoethanol, 100 units $/ \mathrm{ml}$ penicillin, $100 \mu \mathrm{g} / \mathrm{ml}$ streptomycin, $292 \mu \mathrm{g} / \mathrm{ml}$ glutamine (Invitrogen, Carlsbad, CA, USA), and $100 \mathrm{ng} / \mathrm{ml}$ Flt3 ligand (R\&D Systems, Minneapolis, MN, USA). On days 7-9, immature BMpDCs were collected and stimulated with $2 \mu \mathrm{m} \mathrm{CpG-}$ oligodeoxynucleotides (ODN-2216; Hokkaido System Science, Hokkaido, Japan) for 24 hours to induce maturation.

2.4. Chemotaxis Assay. Mature BMpDCs were suspended in modified RPMI 1640 medium (Sigma, St. Louis, MO, USA) containing 1\% FBS and then incubated with each Kampo 
TABLE 1: Components and their percentages in byakkokaninjinto.

\begin{tabular}{lcc}
\hline Components & Full-botanical plant names & Percentage \\
\hline Gypsum Fibrosum & Natural hydrous calcium & 48 \\
Ginseng Radix & Panax ginseng C. A. Meyer & 5 \\
Glycyrrhizae Radix & $\begin{array}{c}\text { Glycyrrhiza uralensis Fischer } \\
\text { Anemarrhenae }\end{array}$ & 6 \\
Rhizoma & Anemarrhena asphodeloides & 16 \\
Oryzae Fructus & Bunge & 25 \\
\hline
\end{tabular}

TABLE 2: 86 kinds of Kampo prescriptions.

\begin{tabular}{|c|c|}
\hline 1 & Anchusan \\
\hline 2 & Inchinkoto \\
\hline 3 & Eppikajutsuto \\
\hline 4 & Orengedokuto \\
\hline 5 & Kakkonto \\
\hline 6 & Kamiuntanto \\
\hline 7 & Kamikihito \\
\hline 8 & Kamishoyosan \\
\hline 9 & Kihito \\
\hline 10 & Keishito \\
\hline 11 & Keishibukuryogan \\
\hline 12 & Goshuyuto \\
\hline 13 & Goreisan \\
\hline 14 & Saikokaryukotsuboreito \\
\hline 15 & Saikokeishito \\
\hline 16 & San'Oshashinto \\
\hline 17 & Sansoninto \\
\hline 18 & Shikunshito \\
\hline 19 & Shimotsuto \\
\hline 20 & Shakuyakukanzoto \\
\hline 21 & Juzentaihoto \\
\hline 22 & Shosaikoto \\
\hline 23 & Shoseiryuto \\
\hline 24 & Shimbuto \\
\hline 25 & Daikenchuto \\
\hline 26 & Daisaikoto \\
\hline 27 & Chotosan \\
\hline 28 & Tokakujokito \\
\hline 29 & Tokishakuyakusan \\
\hline 30 & Ninjinto \\
\hline 31 & Bakumondoto \\
\hline 32 & Hachimijiogan (decoction) \\
\hline 33 & Hangekobokuto \\
\hline 34 & Hangeshashito \\
\hline 35 & Byakkokaninjinto \\
\hline 36 & Boiogito \\
\hline 37 & Bofutsushosan \\
\hline 38 & Hochuekkito \\
\hline 39 & Maoto \\
\hline 40 & Maobushisaishinto \\
\hline 41 & Unkeito \\
\hline 42 & Unseiin \\
\hline 43 & Ogikenchuto \\
\hline 44 & Kambakutaisoto \\
\hline 45 & Kyukikyogaito \\
\hline 46 & Keigairengyoto \\
\hline 47 & Keishikashakuyakuto \\
\hline 48 & Keishikajutsubuto \\
\hline 49 & Keishikaryukotsuboreito \\
\hline
\end{tabular}

TABle 2: Continued.

\begin{tabular}{lc}
\hline 50 & Keishishakuyakuchimoto \\
51 & Kososan \\
52 & Goshajinkigan \\
53 & Goshakusan \\
54 & Saikokeishikankyoto \\
55 & Saikoseikanto \\
56 & Saibokuto \\
57 & Saireito \\
58 & Jiinkokato \\
59 & Shigyakusan (decoction) \\
60 & Shakanzoto \\
61 & Jumihaidokuto \\
62 & Shokenchuto \\
63 & Shofusan \\
64 & Seishinrenshiin \\
65 & Seihaito \\
66 & Sokeikakketsuto \\
67 & Daiokanzoto \\
68 & Daiobotampito \\
69 & Daibofuto \\
70 & Chikujountanto \\
71 & Choijokito \\
72 & Choreito \\
73 & Tokishigyakukagoshuyushokyoto \\
74 & Ninjin'Yoeito \\
75 & Hangebyakujutsutemmato \\
76 & Bukuryoin \\
77 & Heiisan \\
78 & Makyokansekito \\
79 & Makyoyokukanto \\
80 & Yokuininto \\
81 & Yokukansan \\
82 & Rikkunshito \\
83 & Ryutanshakanto \\
84 & Ryokyojutsukanto \\
85 & Ryokeijutsukanto \\
86 &
\end{tabular}

formula extract or herbal medicine extract for 3 hours at $37^{\circ} \mathrm{C}$. Chemotaxis experiments with BMpDCs were performed in an EZ-TAXIScan ${ }^{\mathrm{TM}}$ chamber according to the manufacturer's protocol (GE Healthcare Japan, Tokyo, Japan). A BMpDC suspension $\left(1 \times 10^{6}\right.$ cells $\left./ \mathrm{ml}\right)$ was injected into one side of the chamber, and $1 \mu \mathrm{l}$ of CCL21 $(250 \mu \mathrm{g} / \mathrm{ml})$ was injected into the opposite side of the chamber. A concentration gradient of CCL21 was formed, and the migration of the BMpDCs toward the more concentrated side of a CCL21 gradient was observed. BMpDC migration was recorded with a CCD camera located beneath the chamber every 30 seconds for 1 hour. At the end of the chemotaxis assay, the number of migrated $\mathrm{BMpDCs}$ during 30 minutes and the velocity and directionality of the migrating BMpDCs were analyzed by the TAXIScan Analyzer 2.

2.5. Viability Assay and Analysis of CCR7 Expression Level. Treatment with byakkokaninjinto to mature BMpDCs was performed in the same way as the chemotaxis assay. Mature BMpDCs were suspended in modified RPMI 1640 medium containing $1 \%$ FBS and then incubated with 
byakkokaninjinto for 3 hours at $37^{\circ} \mathrm{C}$. Subsequently, BMpDCs were stained with Via-Probe (Becton Dickinson, San Jose, CA, USA), anti-mouse mPDCA-1-APC (Miltenyi Biotec, Bergisch Gladbach, Germany), and antimouse CD11c-FITC (BD Biosciences, San Jose, CA, USA). To analyze the expression level of CCR7 on BMpDCs, BMpDCs were stained with anti-mouse CD197 (CCR7)PE-Cy7 (eBioscience).

2.6. Flow Cytometry and Antibodies. Mature BMpDCs were suspended in FACS buffer (0.01 M phosphate-buffered saline (PBS) containing $1 \% \mathrm{BSA}$ and $0.2 \% \mathrm{NaN}_{3}$ ) and stained with the following antibodies: anti-mouse mPDCA-1-APC (Miltenyi Biotec), anti-mouse CD11c-FITC (BD Biosciences), anti-mouse MHC class II-PE (eBioscience, San Diego, CA, USA), and anti-mouse CD197 (CCR7)-PE-Cy7 (eBioscience). Cell proportions were analyzed with a $\mathrm{BD}$ FACSCantoII flow cytometer (BD Biosciences).

2.7. DNFB-Induced Allergic Contact Dermatitis Mouse Model. Male BALB/c mice (6 weeks old) were administered intraperitoneally $10 \mu \mathrm{g}$ DNP-OVA with $1 \mathrm{mg}$ aluminum hydrogel on day 0 . Byakkokaninjinto $(0.5 \mathrm{~g} / \mathrm{kg}$ or $1.0 \mathrm{~g} / \mathrm{kg})$ in $0.5 \%$ methylcellulose was administered orally on day 13 . Skin lesions were induced by painting the ear with $0.1 \% 1$ fluoro-2,4-dinitrobenzene (DNFB) in ethanol on day 14. Ear swelling induced with DNFB was evaluated by measuring ear thickness at 1 hour, 2 days, and 7 days after DNFB painting.

2.8. Statistical Analysis. The results are expressed as the mean \pm SEM. Significant differences among groups were evaluated by one-way analysis of variance (ANOVA) followed by Dunnett's test for multiple comparisons, and significant differences between groups were evaluated with an unpaired Student's $t$-test. A $P$ value less than 0.05 was considered significant.

\section{Results}

3.1. Effects of Kampo Formulas on the Migration of $B M p D C s$. To identify drugs that regulate DC migration, 86 Kampo formula extracts were screened using an EZTAXIScan chemotaxis assay. The chemotactic responses of BMpDCs were observed following stimulation with the CCR7 ligand CCL21, and BMpDCs migrated toward the more concentrated side of the CCL21 concentration gradient (Figure 1(a)). Among the 86 Kampo formula extracts, byakkokaninjinto significantly suppressed the migration of BMpDCs from 25 minutes to 30 minutes at each time point (Figures $1(\mathrm{a})$ and $1(\mathrm{~b}), P<0.05$ ), and shimotuto significantly accelerated the migration of BMpDCs (data not shown). The other 84 Kampo formula extracts did not show significant effects on BMpDC migration. Compared with the vehicle, byakkokaninjinto obviously decreased the number of migrated BMpDCs during 30 minutes (Figure 1(b)). In addition, we also performed a detailed analysis of the inhibitory effect of byakkokaninjinto by analyzing the velocity and directionality of BMpDC migration (Figure 1(c)). The velocity of the migration of BMpDCs treated with byakkokaninjinto $(0.08 \pm 0.00 \mu \mathrm{m} / \mathrm{sec})$ was significantly slower than that of $\mathrm{BMpDCs}$ treated with the vehicle $(0.11 \pm 0.00 \mu \mathrm{m} / \mathrm{sec} ; P<0.05)$. In addition, the direction of $\mathrm{BMpDC}$ migration was calculated as the radian of cells migrating toward the more concentrated side of the concentration gradient of CCL21. The directionality of the migration of BMpDCs treated with byakkokaninjinto $(0.48 \pm 0.03 \mathrm{rad})$ was significantly lower than that of those treated with the vehicle $(0.67 \pm 0.03 \mathrm{rad} ; P<0.05)$. These results indicate that byakkokaninjinto has a notable inhibitory effect on pDC migration.

3.2. Viability Assay with BMpDCs Treated with Byakkokaninjinto. To verify that the inhibitory effect of byakkokaninjinto on BMpDC migration is not due to toxic side effects, we examined the viability of $\mathrm{BMpDCs}$ treated with byakkokaninjinto. BMpDCs treated with byakkokaninjinto or the vehicle were stained with Via-Probe, an antiCD11c antibody, and an anti-mPDCA-1 antibody. Dead BMpDCs (CD11 ${ }^{\text {int }}$ mPDCA- ${ }^{+}$Via-Probe ${ }^{+}$) were detected by flow cytometry. The proportion of dead BMpDCs following vehicle treatment was $4.3 \pm 0.5 \%$, and the proportion following byakkokaninjinto treatment was $3.6 \pm 0.2 \%$ (Figure 2). Thus, byakkokaninjinto has no toxic side effects on BMpDCs.

3.3. Expression Level of CCR7 on BMpDCs Treated with Byakkokaninjinto. The maturation of $\mathrm{BMpDCs}$ is accompanied by the upregulation of CCR7 expression, which leads to increased sensitivity to CCL21. Thus, the expression level of CCR7 on BMpDCs was analyzed by flow cytometry. The expression level of CCR7 on BMpDCs treated with byakkokaninjinto was comparable to that on BMpDCs treated with the vehicle (Figure 3), indicating that byakkokaninjinto has no effect on the expression of CCR7 on BMpDCs.

\subsection{Effects of the Components of Byakkokaninjinto on BMpDC} Migration. Byakkokaninjinto is composed of Gypsum Fibrosum, Ginseng Radix, Glycyrrhizae Radix, Anemarrhenae Rhizoma, and Oryzae Fructus. We examined the effect of each component extract on the migration of BMpDCs. BMpDCs were treated with one component of byakkokaninjinto $(0.02 \mathrm{mg} / \mathrm{ml}, 0.05 \mathrm{mg} / \mathrm{ml}$, or $0.1 \mathrm{mg} / \mathrm{ml})$ or the vehicle for 3 hours. The number of BMpDCs chemotaxing towards the CCL21 concentration gradient following treatment with each component extract or vehicle was increased during 30 minutes. At the dose of $0.1 \mathrm{mg} / \mathrm{ml}$, the number of migrated BMpDCs was obviously decreased by the treatment of Gypsum Fibrosum, Ginseng Radix, and Glycyrrhizae Radix (Figure 4(a)) There was no effect of Anemarrhenae Rhizoma and Oryzae Fructus on the number of migrated BMpDCs (Figure 4(a)). To confirm the effect of each component, the value of the velocity and the directionality of BMpDC migration under treatment of each 


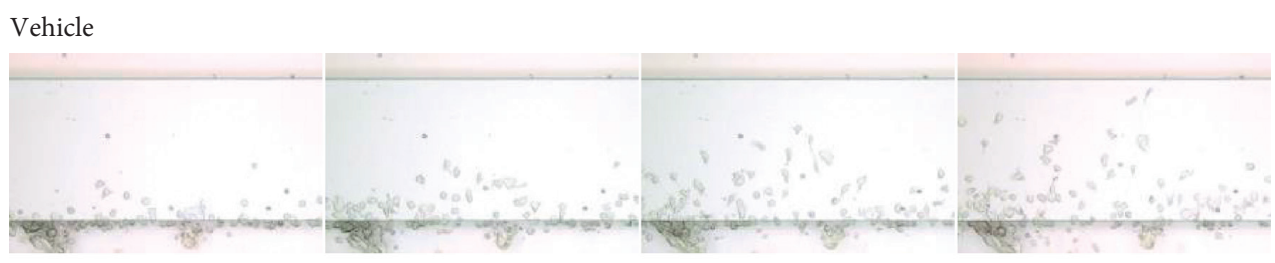

Byakkokaninjinto

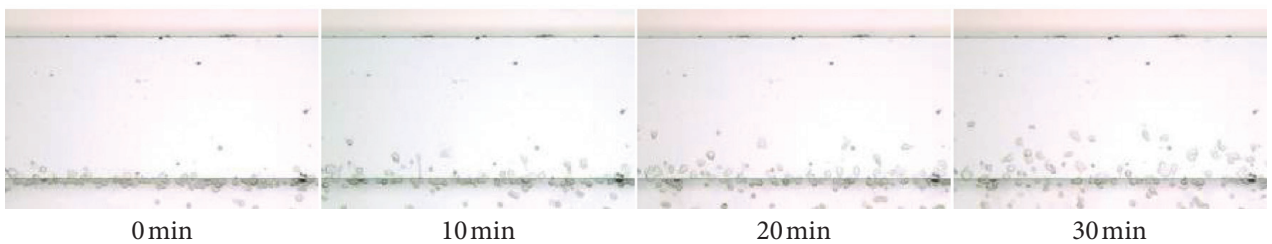

(a)

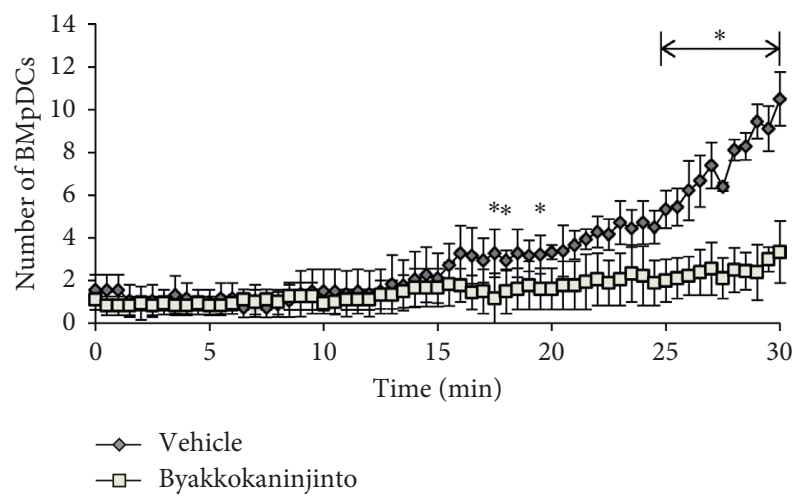

(b)

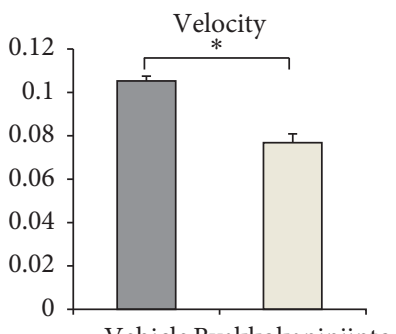

Vehicle Byakkokaninjinto

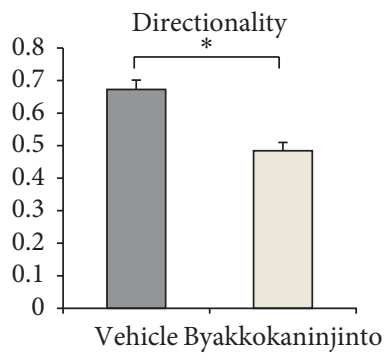

(c)

FIGURE 1: The effects of byakkokaninjinto on the migration of BMpDCs. Chemotactic responses were induced in BMpDCs by stimulation with the CCR7 ligand CCL21. Time-lapse images of the migration of BMpDCs treated with byakkokaninjinto $(0.1 \mathrm{mg} / \mathrm{ml}) \mathrm{or}$ the vehicle were recorded in an EZ-TAXIScan chemotaxis assay. (a) The number of byakkokaninjinto-treated BMpDCs that migrated during 30 minutes is indicated by the line graph and (b) data are expressed as the mean $\pm \mathrm{SE}\left({ }^{*} P<0.05\right.$, vs. the vehicle; $\left.n=3\right)$. The effects of byakkokaninjinto on the velocity and directionality of BMpDC migration are represented by the bar chart; (c) data are expressed as the mean \pm SE ( ${ }^{*} P<0.05$, vs. the vehicle; $n=5$ ).

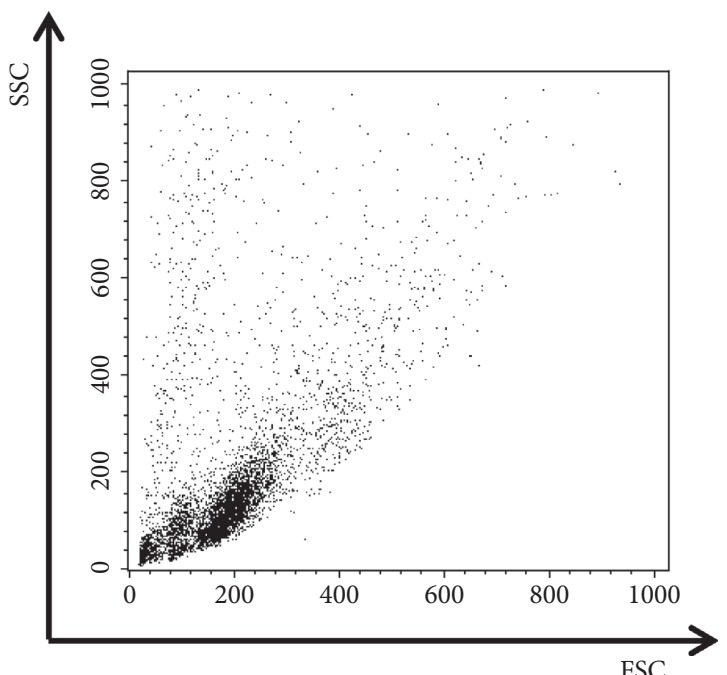

(a)

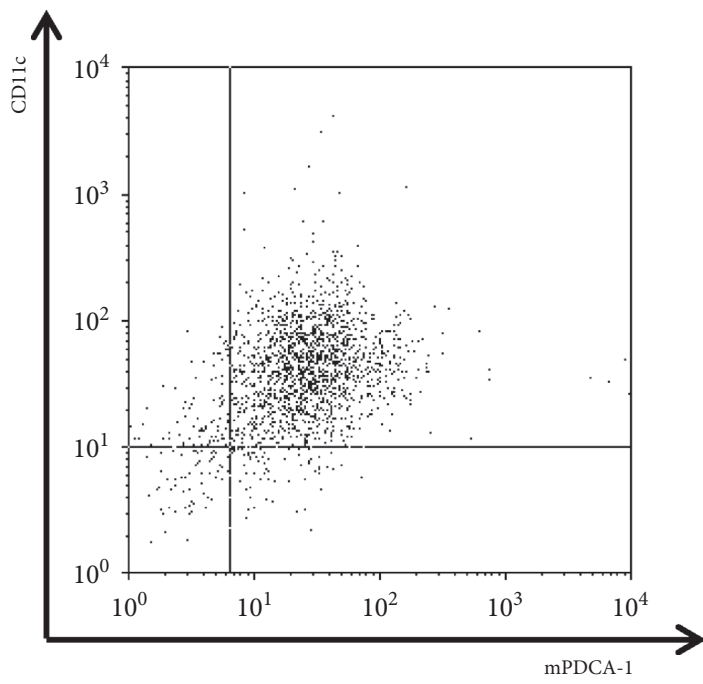

(b)

Figure 2: Continued. 


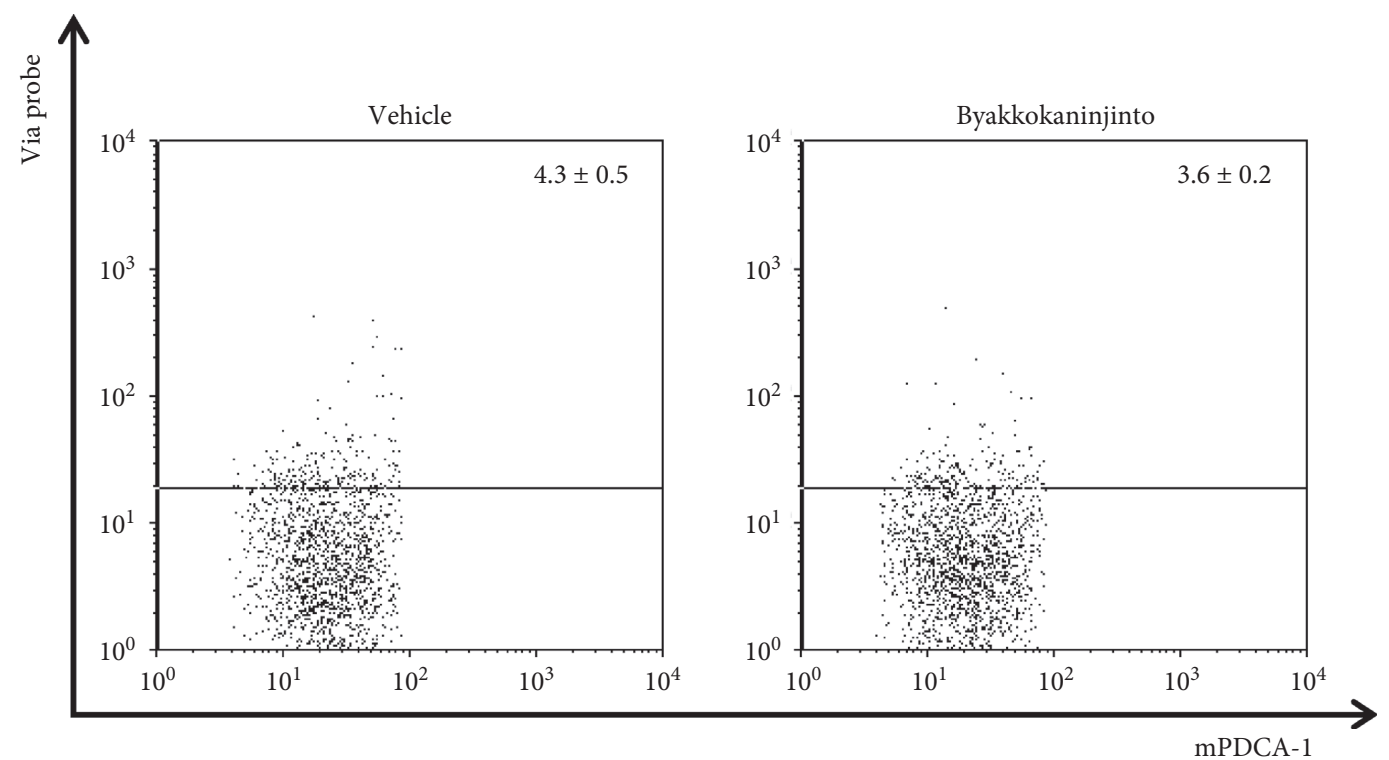

(c)

Figure 2: The detection of cell death caused by byakkokaninjinto. The toxicity of byakkokaninjinto to BMpDCs was detected by flow cytometry. The percentage of dead BMpDCs $\left(\mathrm{CD} 11 \mathrm{c}^{\text {int }}\right.$ mPDCA- $1^{+}$Via-Probe $\left.{ }^{+}\right)$was determined. Dot plots show representative data. Data are expressed as the mean $\pm \mathrm{SE}(n=3)$.

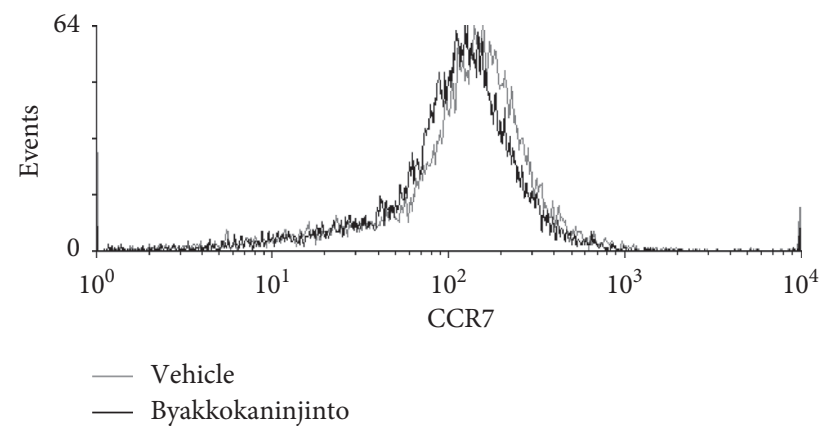

Figure 3: The effects of byakkokaninjinto on CCR7 expression in BMpDCs. The expression of CCR7 on BMpDCs treated with $0.1 \mathrm{mg} / \mathrm{ml}$ byakkokaninjinto (black line) or the vehicle (gray line) was detected by flow cytometry. Representative CCR7 expression data are represented by the line graph.
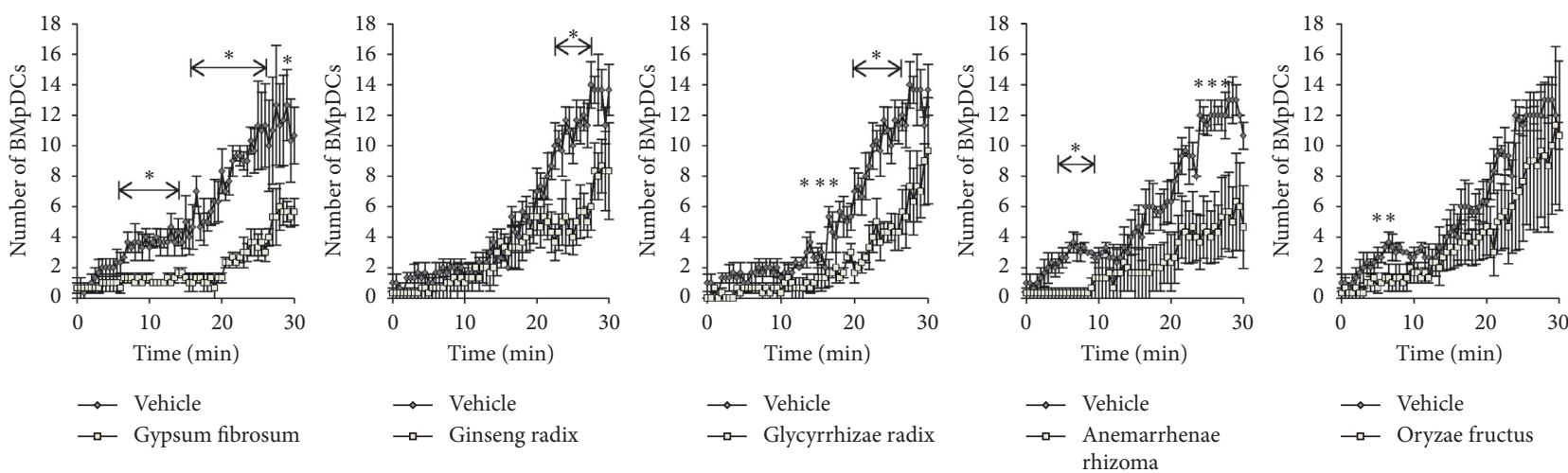

(a)

FIgURE 4: Continued. 


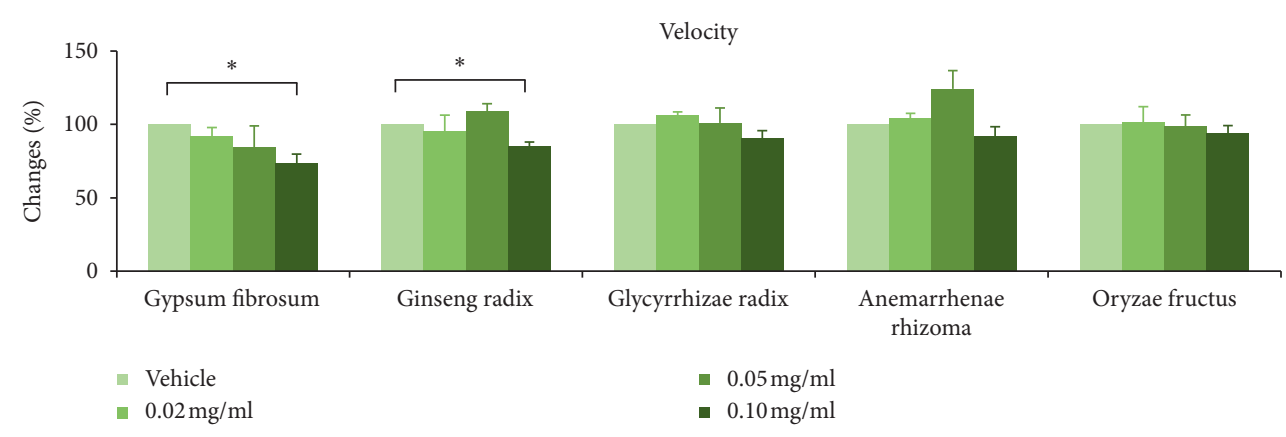

(b)

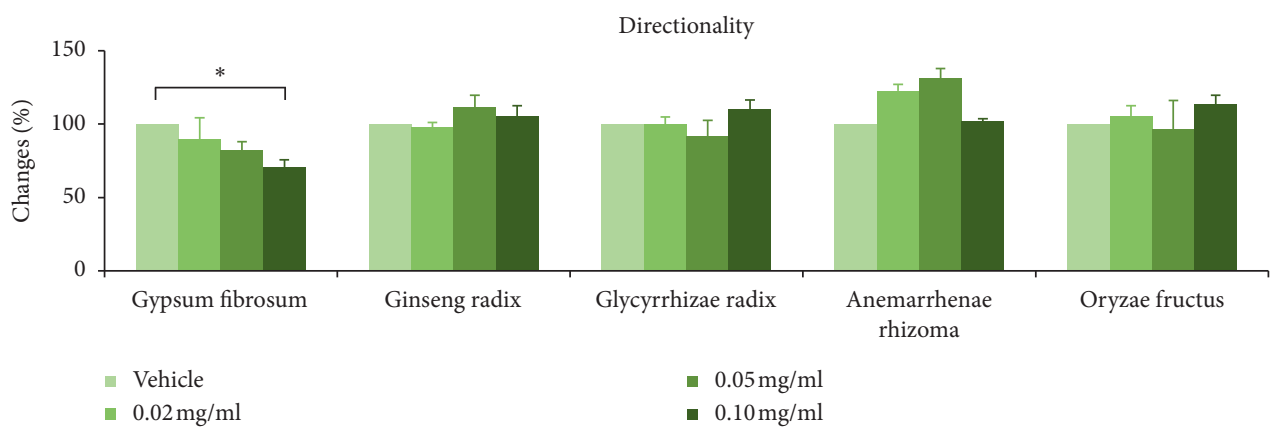

(c)

FIgURE 4: The effects of byakkokaninjinto components on BMpDC migration. The number of migrated BMpDCs with the treatment of each component $(0.10 \mathrm{mg} / \mathrm{ml})$ is indicated by the line graph; (a) data are expressed as the mean $\pm \mathrm{SE} \mathrm{(}{ }^{*} P<0.05$, vs. the vehicle; $\left.n=3\right)$. The effects of each component of byakkokaninjinto at doses of $0.02 \mathrm{mg} / \mathrm{ml}, 0.05 \mathrm{mg} / \mathrm{ml}$, and $0.10 \mathrm{mg} / \mathrm{ml}$ on the velocity and directionality of BMpDC migration were measured by an EZ-TAXIScan chemotaxis assay. The bar chart shows the change rates of BMpDC migration velocity (b) and directionality (c) induced by treatment at each dose versus treatment with the vehicle. Data are expressed as the mean $\pm \mathrm{SE}\left({ }^{*} P<0.05\right.$, vs. the vehicle; $n=3)$.

component of byakkokaninjinto $(0.02 \mathrm{mg} / \mathrm{ml}, 0.05 \mathrm{mg} / \mathrm{ml}$, or $0.1 \mathrm{mg} / \mathrm{ml}$ ) were examined. The velocity of $\mathrm{BMpDC}$ migration in the vehicle treatment group was $0.12 \pm 0.00 \mu \mathrm{m} /$ sec. Treatment with Gypsum Fibrosum $(0.10 \mathrm{mg} / \mathrm{ml})$ or Ginseng Radix $(0.10 \mathrm{mg} / \mathrm{ml})$ for 3 hours significantly reduced the velocity of BMpDC migration by $24.7 \%$ and $13.3 \%$, respectively (Figure $4(\mathrm{~b}) ; P<0.05$ ). In addition, the inhibitory effect of Gypsum Fibrosum was dose-dependent.

The directionality of $\mathrm{BMpDC}$ migration in the vehicle group was $0.62 \pm 0.04 \mathrm{rad}$. Treatment with Gypsum Fibrosum $(0.10 \mathrm{mg} / \mathrm{ml})$ significantly reduced the directionality of BMpDC migration by $37.5 \%(P<0.05)$, and the inhibitory effect of Gypsum Fibrosum was dose-dependent (Figure 4(c)). Therefore, these results indicate that Gypsum Fibrosum is an active component in the inhibitory effect of byakkokaninjinto on BMpDC migration.

\subsection{Effects of Byakkokaninjinto on an DNFB-Induced Allergic} Contact Dermatitis Model. There are several inconsistent reports on the role of pDCs in the onset of dermatitis, which remains controversial. In the present study, we used a murine model of DNFB-induced allergic contact dermatitis that exhibits allergic contact dermatitis symptoms (ear swelling) with an immediate-phase reaction (IPR) at 1 hour, a late-phase reaction (LPR) at 2 days, and a very late-phase reaction (vLPR) at 6-10 days after DNFB administration [21]. Oral administration of byakkokaninjinto exhibited a tendency to suppress ear swelling during the IPR in a dosedependent manner and then markedly prevented ear swelling during the LPR. However, byakkokaninjinto had no effect on ear swelling during the vLPR (Figure 5).

\section{Discussion}

The present study revealed that byakkokaninjinto inhibits the migration of BMpDCs and that Gypsum Fibrosum, one of the components of byakkokaninjinto, has a potent inhibitory effect on BMpDC migration. Furthermore, it suggested that pDCs are involved in the onset of allergic contact dermatitis and that byakkokaninjinto suppresses the onset of allergic contact dermatitis by inhibiting pDC migration.

Byakkokaninjinto significantly inhibited the migration of BMpDCs without inducing cellular toxicity in BMpDCs or enhancing the expression of CCR7 on BMpDCs. These results indicated that the inhibitory effect of byakkokaninjinto on BMpDC migration was caused by the inhibition of CCR7 signaling cascades. The activation of the CCR7 signaling pathway elicited by CCL21 is related to trimeric GTP-binding proteins, Jak family proteins, and Rho family proteins [3]. Rho, Rac, and Cdc42, members of the Rho family of proteins, play crucial roles in DC migration [22]. In particular, it has been reported that $\mathrm{BMpDCs}$ derived from DOCK2-deficient mice hardly migrate toward the more concentrated side of a concentration gradient of CCL21 [20]. Furthermore, Rac1 activation induced by CCL21 is almost 


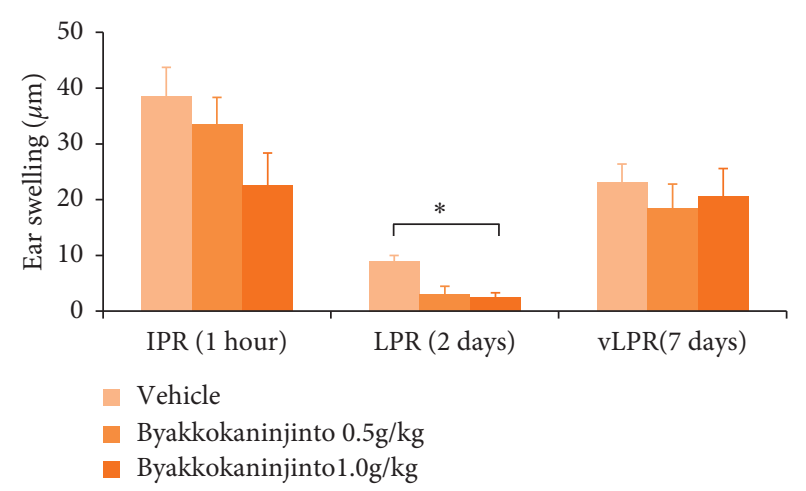

FIgUre 5: The effects of byakkokaninjinto on DNFB-induced allergic contact dermatitis model. Byakkokaninjinto $(0.5 \mathrm{~g} / \mathrm{kg}$ or $1.0 \mathrm{~g} / \mathrm{kg}$ ) was given by oral administration to DNFB-induced allergic contact dermatitis model mice 1 day before DNFB sensitization. Ear swelling was monitored during the IPR (1 hour), LPR (2 days), and vLPR (7 days). The bar chart shows the ear thicknesses of mice treated with $0.5 \mathrm{~g} / \mathrm{kg}$ or $1.0 \mathrm{~g} / \mathrm{kg}$ byakkokaninjinto or the vehicle during the IPR, LPR, and vLPR. Data are expressed as the mean \pm SE ( ${ }^{*} P<0.05$, vs. the vehicle; $\left.n=5\right)$.

abolished in DOCK2-deficient BMpDCs, although wild-type BMpDCs exhibit Rac1 activation [20]. Accordingly, Rho family proteins, including Rac1-DOCK2, are essential in the migration of pDCs. Thus, it is assumed that byakkokaninjinto inhibits the signaling pathway of Rac1-DOCK2 to suppress pDC migration.

We investigated the effect of each component of byakkokaninjinto on BMpDC migration and demonstrated that Gypsum Fibrosum and Ginseng Radix have inhibitory effects on BMpDC migration. However, the effective doses $(0.1 \mathrm{mg} / \mathrm{ml})$ of Gypsum Fibrosum and Ginseng Radix were higher than the amount contained in byakkokaninjinto. Moreover, makyokansekito, which contains the same dose of Gypsum Fibrosum as byakkokaninjinto, showed no inhibitory effect on the migration of pDCs. In addition, a high dose of Ginseng Radix is contained in many Kampo formulas, such as daikenchuto and shikunshito. However, these Kampo formulas had no effect on the migration of pDCs. In general, the pharmacological effects of Kampo formulas containing many herbal components are characterized by synergistic effects or additive effects of multiple herbal components and thus are complicated [23]. These results suggest that the inhibitory effect of byakkokaninjinto on pDC migration is attributed to the synergistic effects of multiple herbal medicines in byakkokaninjinto, including Gypsum Fibrosum and Ginseng Radix.

It has been reported that pDCs highly infiltrate the lesional skin of patients with contact dermatitis $[8,24]$ or atopic dermatitis $[9,10]$, whereas the proportion of $\mathrm{CD} 304^{+}$ $\mathrm{BDCA}^{+} \mathrm{pDCs}$ does not change in the peripheral blood mononuclear cell population [9]. Conversely, $\mathrm{BDCA} 2^{+} \mathrm{pDCs}$ are not recruited into the lesional skin of patients with atopic dermatitis [24, 25]. Even in patients with atopic dermatitis, the proportion of $\mathrm{CD} 123^{+}$pDCs in the blood is higher than that in normal subjects [10]. In ovalbumin-induced dermatitis model mice, Wang et al. reported that the infiltration of pDCs was increased in the inflamed skin and that treatment with the immunostimulatory sequence $\mathrm{CpG}$ reduced $\mathrm{pDC}$ infiltration and skin inflammation [26], suggesting that pDCs play pivotal roles in the onset and development of dermatitis. However, in DNFB-induced dermatitis model similar to our used model, it is also reported that $\mathrm{pDCs}$ prevent the ear swelling response by inducing systemic tolerance $[27,28]$. Accordingly, the role and distribution of pDCs in dermatitis remain poorly understood. In this study, we demonstrated that byakkokaninjinto had an inhibitory effect on pDC migration and suppressed the initiation of DNFB-induced allergic contact dermatitis. Therefore, the migration of pDCs to inflamed sites or the lymph nodes contributes to the onset and development of allergic contact dermatitis.

pDCs are well known for their capacity to present antigens [29-31] and secrete type 1 IFN in response to viral infections $[2,29]$. Acquired immune responses are carried out through $\mathrm{pDC}$ migration to inflamed sites and mature $\mathrm{pDC}$ migration from the inflamed sites to the lymph nodes, where they perform antigen presentation. Consequently, the inhibition of pDC migration by byakkokaninjinto may have a beneficial effect on allergic dermatitis via the suppression of immune responses.

\section{Conclusions}

In conclusion, byakkokaninjinto possesses the ability to inhibit the migration of pDCs, thereby ameliorating allergic dermatitis. Moreover, byakkokaninjinto is anticipated to be used as a therapeutic agent for pDC-related diseases, such as allergic dermatitis.

\section{Data Availability}

The data used to support the findings of this study are included within the article and are available from the corresponding author upon request.

\section{Conflicts of Interest}

The authors declare that there are no conflicts of interest regarding the publication of this article.

\section{Authors' Contributions}

TY and YZ designed the study and wrote the original draft. TY, YZ, AK, SH, and MK performed the experiments and data curation. TY and MK performed and supervised the work. All the authors read and approved the final manuscript.

\section{Acknowledgments}

This study was supported by the JSPS KAKENHI (Grant no. JP19K07908) and 2019 Director Leadership Expenses, Institute of Natural Medicine, University of Toyama.

\section{Supplementary Materials}

Supplementary Figure 1: chemical profile of byakkokaninjinto analyzed by 3D-HPLC. Supplemental Table 1: components of 86 kinds of Kampo prescriptions. Supplemental Table 2: the 
details of herbal extracts in 86 kinds of Kampo prescriptions. (Supplementary Materials)

\section{References}

[1] C. L. Sokol and A. D. Luster, "The chemokine system in innate immunity," Cold Spring Harbor Perspectives in Biology, vol. 7, no. 5, Article ID a016303, 2015.

[2] M. Swiecki and M. Colonna, "The multifaceted biology of plasmacytoid dendritic cells," Nature Reviews Immunology, vol. 15, no. 8, pp. 471-485, 2015.

[3] M. A. Hauser and D. F. Legler, "Common and biased signaling pathways of the chemokine receptor CCR7 elicited by its ligands CCL19 and CCL21 in leukocytes," Journal of Leukocyte Biology, vol. 99, no. 6, pp. 869-882, 2016.

[4] X. Liu, P. Mishra, S. J. Yu et al., "Tolerance induction towards cardiac allografts under costimulation blockade is impaired in CCR7-deficient animals but can be restored by adoptive transfer of syngeneic plasmacytoid dendritic cells," European Journal of Immunology, vol. 41, no. 3, pp. 611-623, 2011.

[5] S. Beckmann, L. Oberdörfer, R. K. Hyde et al., "CCR7 essentially contributes to the homing of plasmacytoid dendritic cells to lymph nodes under steady-state as well as inflammatory conditions," The Journal of Immunology, vol. 186, no. 6, pp. 3364-3372, 2011.

[6] D. Hoff, M. Kurban, and O. Abbas, "Update on the role of plasmacytoid dendritic cells in inflammatory/autoimmune skin diseases," Experimental Dermatology, vol. 25, no. 6, pp. 415-421, 2016.

[7] M. D. Ah Kioon, C. Tripodo, D. Fernandez et al., "Plasmacytoid dendritic cells promote systemic sclerosis with a key role for TLR8," Science Translational Medicine, vol. 10, no. 423, Article ID eaam8458, 2018.

[8] N. Garzorz-Stark, F. Lauffer, L. J. Krause et al., "Toll-like receptor $7 / 8$ agonists stimulate plasmacytoid dendritic cells to initiate TH17-deviated acute contact dermatitis in human subjects," Journal of Allergy and Clinical Immunology, vol. 141, no. 4, pp. 1320-1333, 2018.

[9] M. C. Thomas, T. M. M. Van Capel, J. D. Bos, E. F. Knol, M. L. Kapsenberg, and E. C. De Jong, "Aberrant function of peripheral blood myeloid and plasmacytoid dendritic cells in atopic dermatitis patients," Journal of Allergy and Clinical Immunology, vol. 122, no. 5, pp. 969-976, 2008.

[10] H. Hashizume, T. Horibe, H. Yagi, N. Seo, and M. Takigawa, "Compartmental imbalance and aberrant immune function of blood CD123+ (plasmacytoid) and CD11c+ (myeloid) dendritic cells in atopic dermatitis," The Journal of Immunology, vol. 174, no. 4, pp. 2396-2403, 2005.

[11] P.-L. Wang and A. Kaneko, "Introduction to Kampo medicine for dental treatment-oral pharmacotherapy that utilizes the advantages of Western and Kampo medicines," Japanese Dental Science Review, vol. 54, no. 4, pp. 197-204, 2018.

[12] S. Takayama, R. Arita, A. Kikuchi, M. Ohsawa, S. Kaneko, and T. Ishii, "Clinical practice guidelines and evidence for the efficacy of traditional Japanese herbal medicine (Kampo) in treating geriatric patients," Frontiers in Nutrition, vol. 5, p. 66, 2018.

[13] P.-Y. Lin, C.-H. Chu, F.-Y. Chang, Y.-W. Huang, H.-J. Tsai, and T.-C. Yao, "Trends and prescription patterns of traditional Chinese medicine use among subjects with allergic diseases: a nationwide population-based study," World Allergy Organization Journal, vol. 12, no. 2, Article ID 100001, 2019.
[14] Z. Wang, J. Cai, Q. L. Fu et al., “Anti-inflammatory activities of compounds isolated from the rhizome of anemarrhena asphodeloides," Molecules, vol. 23, no. 10, p. 2631, 2018.

[15] K. Cheng, J. Kim, and H. Min, "Ginseng, the natural effectual antiviral: protective effects of Korean red Ginseng against viral infection," Journal of Ginseng Research, vol. 40, no. 4, pp. 309-314, 2016.

[16] V. Karuppagounder, M. Nomoto, and K. Watanabe, “Antiinflammatory effects of Kampo medicines in atopic dermatitis," in Japanese Kampo Medicines for the Treatment of Common Diseases: Focus on Inflammation, S. Arumugam and K. Watanabe, Eds., Elsevier, New York, NY, USA, pp. 89-95, 2017.

[17] T. Shimizu, "Efficacy of Kampo medicine in treating atopic dermatitis: an overview," Evidence-Based Complementary and Alternative Medicine, vol. 2013, Article ID 260235, 5 pages, 2013.

[18] C. Tohda, H. Sugahara, Y. Kuraishi, and K. Komatsu, "Inhibitory effect of Byakko-ka-ninjin-to on itch in a mouse model of atopic dermatitis," Phytotherapy Research, vol. 14, no. 3, pp. 192-194, 2000.

[19] T. Tatsumi, T. Yamada, H. K. Nagai, T. Tani, S. Nunome, and I. Saiki, "A Kampo formulation. Byakko-ka-ninjin-to (BaiHu-Jia-Ren-Sheng-Tang) inhibits IgE-mediated triphasic skin reaction in mice. The role of its constituents in expression of the efficacy," Biological \& Pharmaceutical Bulletin, vol. 24, no. 3, pp. 284-290, 2001.

[20] K. Terasawa, Y. Tanaka, A. A. Nishikimi et al., "Differential requirement for DOCK2 in migration of plasmacytoid dendritic cells versus myeloid dendritic cells," Blood, vol. 111, no. 6, pp. 2973-2976, 2008.

[21] K. Inayoshi, M. Masuda, S. Naruto, K. Murata, and H. Matsuda, "Antiallergic activity of unripe Citrus hassaku fruits extract and its flavanone glycosides on chemical substance-induced dermatitis in mice," Journal of Natural Medicines, vol. 63, no. 4, pp. 443-450, 2009.

[22] A. J. Ridley, "Rho GTPase signalling in cell migration," Current Opinion in Cell Biology, vol. 36, pp. 103-112, 2015.

[23] X. Zhou, S. W. Seto, D. Chang et al., "Synergistic effects of Chinese herbal medicine: a comprehensive review of methodology and current research," Frontiers in Pharmacology, vol. 7, p. 201, 2016.

[24] A. Wollenberg, S. Günther, M. S. Moderer et al., "Plasmacytoid dendritic cells: a new cutaneous dendritic cell subset with distinct role in inflammatory skin diseases," Journal of Investigative Dermatology, vol. 119, no. 5, pp. 1096-1102, 2002.

[25] S. Vittorakis, K. Samitas, S. Tousa et al., "Circulating conventional and plasmacytoid dendritic cell subsets display distinct kinetics during in vivo repeated allergen skin challenges in atopic subjects," BioMed Research International, vol. 2014, Article ID 231036, 14 pages, 2014.

[26] G. Wang, N. Fyhrquist-Vanni, H. M.-C. Wolff et al., "Immunostimulatory sequence $\mathrm{CpG}$ elicits Th1-type immune responses in inflammatory skin lesions in an atopic dermatitis murine model," International Archives of Allergy and Immunology, vol. 147, no. 1, pp. 41-51, 2008.

[27] A. Dieu-Nosjean, B. Dubois, H. G. Gheit et al., "Plasmacytoid dendritic cells mediate oral tolerance," Immunity, vol. 29, no. 3, pp. 464-475, 2008.

[28] B. Joubert, G. Joubert, M. Gomez de Agüero, M. Gouanvic, A. Goubier, and D. Kaiserlian, "Sequential role of plasmacytoid dendritic cells and regulatory T cells in oral tolerance," Gastroenterology, vol. 137, no. 3, pp. 1019-1028, 2009. 
[29] B. Reizis, "Plasmacytoid dendritic cells: development, regulation, and function," Immunity, vol. 50, no. 1, pp. 37-50, 2019.

[30] H. Suzuki, K. Jounai, K. Ohshio, T. Fujii, and D. Fujiwara, "Administration of plasmacytoid dendritic cell-stimulative lactic acid bacteria enhances antigen-specific immune responses," Biochemical and Biophysical Research Communications, vol. 503, no. 3, pp. 1315-1321, 2018.

[31] J. A. Villadangos and L. Young, "Antigen-presentation properties of plasmacytoid dendritic cells," Immunity, vol. 29, no. 3, pp. 352-361, 2008. 